yearly trend in the percentage of the population with body mass index (BMI) in normal-weight (BMI<25), over-weight (BMI 25-29.9), and obese (BMI>30) ranges, is modelled using multinomial logistic regression, stratifying by gender and age group $(20-39,40-59,>60$ years). The fitted models are used to compare trends between states and to forecast levels of obesity in the future. A previously developed micro-simulation model is used to assess the burden of 13 diseases caused by obesity and estimate the economic impact implied by the forecasted trends. Data of the US-National Health and Nutrition Examination Survey collected over the same period are used to investigate the extent of self-reporting bias in BRFSS.

Results In 2010, the proportion of obese (BMI>30) men and women in the US was $33 \%$ and $35 \%$, respectively, whilst the proportions of over-weight (BMI 25-30) were $42 \%$ and $34 \%$. The fitted models forecast an increase in the proportion of obese individuals to up to $70 \%$ by 2030 . The results reveal increasing levels of obesity in all states, although the rate of increase varies among states. Comparing BRFSS data with data from NHANES, showed that at all ages both men and women slightly over-report their height, whilst women under-report their weight by $5 \mathrm{~kg}$ on average. Under-reporting of weight is reduced after age 65.

Conclusion Obesity rates are rapidly and steadily increasing in the US posing a threat to population health and a substantial economic burden. As self-reporting bias may significantly underestimate BMI in women, the estimated burden of obesity may be conservative.

\section{OP27 DIETARY FIBRE INTAKE AND FATAL STROKE RISK IN THE UK WOMEN'S COHORT STUDY}

doi:10.1136/jech-2012-201753.027

'DE Threapleton, 'VJ Burley, ${ }^{2} \mathrm{DC}$ Greenwood, 'JE Cade. 'Nutritional Epidemiology Group, University of Leeds, Leeds, UK; ${ }^{2}$ Biostatistics Unit, University of Leeds, Leeds, UK

Background In the UK in 2008, stroke was responsible for over 43,000 deaths. Dietary fibre intake may reduce stroke risk through modifying one or more risk factor such as body weight, blood cholesterol, or blood pressure. Food sources of fibre can be examined in relation to risk as different foods contain different types of fibre which have differing proposed actions within the body.

Methods 31,036 women recruited in 1995 provided dietary information relating to the previous 12 months using a 217-item food frequency questionnaire. Total dietary fibre intakes were estimated as both non-starch polysaccharide (NSP) and Association of Official Analytical Chemist (AOAC) values. Insoluble fibre, soluble fibre and fibre (NSP) from a variety of food sources were also examined. Stroke mortality was registered from baseline through the Office of National Statistics. Cox proportional hazard ratios were generated to explore risk using both categorical (fifths of intake) and continuous exposure variables. Model adjustments: age, body mass index, energy intake, ethanol, physical activity, smoking and socio-economic status.

Results After 14 years, 130 fatal stroke cases were observed. Fatal stroke risk was similar for total fibre assessed either as NSP or AOAC and both were protectively associated with risk. Overall, risk was roughly halved in each intake category compared to the lowest although this was only significant in the $3 \mathrm{rd}$ (NSP/AOAC) and 4th (AOAC) category. Those consuming 24 vs. $14 \mathrm{~g} / \mathrm{d}$ of NSP or $37 \mathrm{vs}$. $22 \mathrm{~g} / \mathrm{d}$ AOAC had their risk of stroke halved [Englyst: hazard ratio 0.47 (95\% confidence interval $0.24,0.92) \mathrm{p}=0.028$ ] [AOAC: 0.47 $(0.25,0.89) \mathrm{p}=0.021]$. For fibre from nuts and seeds, the highest intake category $(0.85 \mathrm{~g} / \mathrm{d})$ saw a $56 \%$ risk reduction $[0.44(0.23$, 0.83), $\mathrm{p}=0.012$ ] compared to the lowest $(0 \mathrm{~g} / \mathrm{d})$. Fibre from fruit was also protectively associated with risk of stroke in the $4 \mathrm{th}(5.8 \mathrm{~g} / \mathrm{d}) \mathrm{vs}$. 1 st category $(1.4 \mathrm{~g} / \mathrm{d})[0.50(0.25,0.97), \mathrm{p}=0.041]$. These significant associations were not seen in every category comparison and results for continuous exposures were not statistically significant for any of the exposures explored. No association was observed with fibre from cereals, vegetables or legumes.
Conclusion Results suggest that fibre from certain food sources and not others may be more beneficial for prevention of stroke. This may occur because different foods contain fibre fractions in varying quantities. Non-significant results for the continuous exposures suggest associations may be non-linear. The results may also reflect benefits simply from 'fruit' or 'nut and seed' consumption rather than specifically fibre from these sources.

\section{OP28 DIET QUALITY AND BLOOD PRESSURE IN MIDDLE-AGED MEN AND WOMEN}

doi:10.1136/jech-2012-201753.028

J Harrington, AP Fitzgerald, PM Kearney, VJC McCarthy, G Browne, IJ Perry. Epidemiology \& Public Health, University College Cork, Cork, Ireland

Background Findings from both observational and experimental studies (including the DASH Trial -Dietary Approaches to Stop Hypertension) are consistent with a significant, causal role for dietary salt intake in the distribution of blood pressure (BP) in populations. The DASH diet quality score, based on the intervention arm in the DASH-trial, has emerged as a potentially useful measure of diet quality in adult populations. We have studied associations between DASH score and blood pressure, both clinic and 24 hour ambulatory measurements (ABPM) in middle-aged men and women. Methods We used cross-sectional data from two studies of men and women aged 50 to 69 years, recruited in $1998(n=1018)$ and 2010 $(\mathrm{n}=2047)$. Participants completed a physical examination including three standardised clinical BP recordings and a general health and lifestyle questionnaire. A sub-sample $(\mathrm{n}=1189)$ in $2010 \mathrm{had} 24 \mathrm{hr}$ ambulatory BP measurements (ABPM). Diet quality was assessed using a DASH score constructed from a standardised Willett FFO. DASH scores were categorised into quintiles, with lower quintiles indicating less healthy diets. Hypertension was defined as clinic BP $>140 / 90 \mathrm{mmHg}$ (mean of readings 2 and 3 ) and 24-hour ABPM $>130 / 70 \mathrm{mmHg}$.

Results Clear inverse trends were seen between DASH scores and systolic (SBP) and diastolic (DBP) BP in clinic and ABPM recordings. The associations between DASH score and clinic BP were similar in the 1998 and 2010 datasets. In the 2010 data, clinic SBP increased by $7.5 \mathrm{mmHg}$ in men and $5.1 \mathrm{mmHg}$ in women between the highest and lowest DASH quintiles and 24-hour ABPM systolic BP increased by $6.3 \mathrm{mmHg}$ and $5.4 \mathrm{mmHg}$ in men and women respectively between the highest and lowest DASH quintiles. In fully adjusted analyses, the odds ratios (OR) for clinic hypertension and ABPM hypertension in participants with DASH score in the first relative to the fifth DASH score quintile were as follows: clinic hypertension: OR 1.60 (95\% CI 0.9-2.8), ABPM hypertension: OR 4.2; $95 \% \mathrm{CI}$ [1.1-15.9]). Stratifying by gender, these trends persisted for men however they were attenuated for women.

Conclusion This study provides evidence of criterion validity for the use of DASH score as a measure of diet quality, especially in the diet-hypertension relationship in men. Our findings are consistent with the hypothesis that dietary patterns exert effects beyond the sum of the component parts. Public policy promoting a DASH-style healthy diet could have a significant impact on population health by reducing average blood pressure in the population.

\section{Socioeconomic Inequalities II}

\section{OP29 ETHNIC AND SOCIOECONOMIC INFLUENCES ON CHILDHOOD BLOOD PRESSURE: THE CHILD HEART AND HEALTH STUDY IN ENGLAND (CHASE)}

doi:10.1136/jech-2012-201753.029

C Thomas, CM Nightingale, A Donin, AR Rudnicka, CG Owen, D Cook, PH Whincup. Division of Population Health Sciences and Education, St Georges University of London, London, UK 